\title{
Les mots d'emprunt et les transferts culturels : l'influence du français sur le suédois
}

Đurđica Hruškar et Karl Gadelii

Université Paris-Sorbonne

La langue suédoise a été influencée par diverses langues à des époques différentes. La langue française, toute comme la culture française, figurent parmi celles ayant exercé une influence considérable, en laissant de nombreuses traces dans le lexique de la langue suédoise contemporaine.

Les questions principales auxquelles nous nous attacherons à répondre concernent la manière dont la langue française, et éventuellement la culture française, sont présentes dans la langue suédoise de nos jours, de quelles manières elle se manifestent et quelles sont les voies de leur introduction, ainsi que leurs domaines d'influence. Nous allons, tout d'abord, résumer l'histoire de l'influence lexicale et culturelle exercée par le français sur le suédois, en citant des exemples tirés

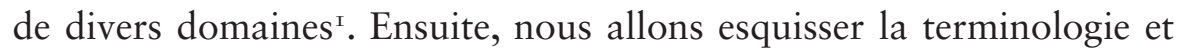
la typologie principale de l'emprunt lexical. Finalement, nous chercherons à cerner l'amplitude de l'influence exercée par le français sur le suédois de nos jours, et cela en procédant à trois tâches : premièrement, en analysant les listes de nouveaux mots de la langue suédoise, deuxièmement, en testant la prononciation de quelques anciens emprunts au français auprès des suédophones natifs et, troisièmement, en étudiant des mots d'origine française dans des secteurs sensibles à l'image de marque française.

\section{Les contacts franco-suédois et les emprunts lexicaux français en suédois : un aperçu historique}

Les premiers contacts franco-scandinaves commencent avec la présence des Vikings en France, notamment au long du IX ${ }^{\mathrm{e}}$ siècle, dont les traces

\footnotetext{
How to cite this book chapter:

Hruškar, Đ. et Gadelii, K. 20I 5. Les mots d'emprunt et les transferts culturels : l'influence du français sur le suédois. In: Cedergren, M. et Briens, S. (eds.) Médiations interculturelles entre la France et la Suède. Trajectoires et circulations de 1945 à nos jours. Pp. 262-280. Stockholm: Stockholm University Press. DOI: http://dx.doi. org/IO.I6993/bad.t. License: CC-BY
} 
sont présentes surtout dans le vocabulaire maritime français et dans la toponymie normande. Durant l'époque médiévale, des contacts sont établis avec l'arrivée des moines de l'Abbaye de Clairvaux en Suède au $\mathrm{XII}^{\mathrm{e}}$ siècle et des architectes français (Hallén, 200I : I9). Au XIII ${ }^{\mathrm{e}}$ et $\mathrm{XIV}^{\mathrm{e}}$ siècles, les étudiants suédois poursuivent leurs études en France.

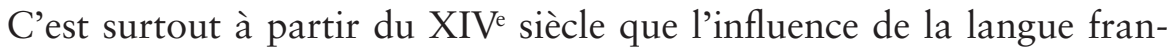
çaise sur le lexique suédois commence à devenir plus importante, p. ex. dans le vocabulaire de la chevalerie (baner "bannière ", kär " amoureux "; Hallén, 200I : I $\left.9^{2}\right)$. Au cours du XVIe siècle, les mots français proviennent souvent du domaine militaire (patron, furir ${ }^{3}$ ), empruntés parfois directement ou bien via l'allemand. Au cours du XVII ${ }^{e}$ siècle, les liens franco-suédois sont renforcés pendant la période de règne de Gustave II Adolphe, grâce au contexte politique et militaire favorable, tout comme durant la période de la reine Christine, où le français est utilisé à la cour de Suède (Engwall, I994: 53 ).

C'est notamment à partir de la deuxième moitié du XVIIe siècle que les mots français sont empruntés de manière plus importante, grâce aux liens politiques et culturels directs entre la France et la Suède, renforcés par la domination culturelle de la France pendant le règne de Louis XIV. Les artistes et les scientifiques français séjournent en Suède, ainsi que de nombreux huguenots, ce qui contribue au renforcement des liens entre les deux pays (Bergman, I972 : I29). De nombreux mots français de cette période entrent par le biais de l'allemand ou du néerlandais, d'où la difficulté de déterminer avec certitude l'origine de l'influence (Bergman, I972 : I30). Parmi les emprunts du XVII siècle, citons ceux du domaine architectural (balkong " balcon », allé " allée »), du domaine des affaires (assurans " assurance ", kredit " crédit»), des accessoires et articles de toilette (puder " poudre ", peruk " perruque »), de l'alimentation et de la cuisine (sås " sauce ", kastrull " casserole »), de la politique et diplomatie (ambassad " ambassade », departement " département ») ou bien encore du domaine artistique (porträtt "portrait », staty « statue » $)^{4}$.

Les contacts culturels sont renforcés aussi dans le domaine du théâtre à partir du règne de Charles XII, avec l'arrivée d'une troupe de théâtre française à la fin du XVII siècle, ainsi que durant le XVIII ${ }^{e}$ siècle, où de nombreuses pièces françaises sont jouées par des troupes diverses (Engwall, I994 : 54). Le journal bihebdomadaire en français Stockholm Gazette couvre également des événements en France (ibid.).

Malgré cette influence remarquable, ce n'est que pendant le règne de Gustave III de la deuxième moitié du XVIII' siècle que l'influence de la 
culture française culmine, en favorisant l'emprunt lexical dans le domaine des arts, du théâtre et de la littérature. Un grand nombre de mots entre grâce à la lecture des textes littéraires français mais aussi par le biais des contacts personnels (Bergman, I972: I 50). La culture française continue à influencer le domaine artistique et la langue sert d'inspiration pour des écrivains suédois dont certains n'hésitent pas à mélanger le suédois et le français, souvent pour parodier l'emploi du français de l'époque. Évoquons ici le poète C. M. Bellman, connu pour ses mélanges linguistiques savoureux (Engwall, I994:56 ; Andersson, I999 : I 5), ou bien A. Strindberg au siècle suivant avec ses emplois du vocabulaire et des expressions françaises riches et variés (Engwall, I994 : 59-6I), tout comme des néologismes créés suivant des règles de formation des mots sur le modèle du vocabulaire emprunté (Ståhle, I962 : 5I-52). L'influence de la langue française ne s'est pourtant pas limitée aux milieux aristocratiques et le vocabulaire est introduit aussi parmi la population rurale via la noblesse provinciale (Hallén, 200I : 20)

À titre d'exemple d'emprunts de cette époque citons ceux du domaine d'habitation et du décor (fåtölj «fauteuil ", garderob " garderobe »), du domaine vestimentaire (kalsonger " caleçon ", uniform " uniforme »), de l'alimentation (glass " glace », maräng " meringue »), du théâtre (ridå « rideau ", pjäs " pièce (de théâtre) »), des arts (karikatyr « caricature ", silhuett / siluett ${ }^{6}$ " silhouette »), de la presse (affisch " affiche ", annons " annonce »), de l'entreprise (direktör " directeur », fabrik « usine, fabrique »), du vocabulaire de société (adjectifs populär " populaire ", au sens " connu de tous ", charmant " charmant»), ou même des prénoms (Sophie, Marie, Emil) .

Malgré le fait que l'influence lexicale française commence à diminuer à partir de la seconde moitié du XIX ${ }^{\mathrm{e}}$ siècle, le français reste toujours une langue donneuse de vocabulaire étranger, tout comme l'allemand et surtout l'anglais qui commence son influence déjà à partir du XIX ${ }^{\mathrm{e}}$ siècle (Edlund \& Hene, I 996 : 57). Parmi les emprunts du XIX siècle citons ceux du domaine vestimentaire et des accessoires (blus "blouse ", brosch "broche »), du domaine culinaire (puré " purée ", omelett " omelette ", charkuteri « charcuterie») ou encore politique (socialism "socialisme ", byråkrati " bureaucratie ", diplomat « diplomate») $)^{8}$.

À l'époque actuelle, l'influence de la culture française par le biais de l'emprunt lexical semble se poursuivre mais dans une moindre mesure. L'influence est notamment visible dans les domaines tels que la restauration et la mode, où les noms français sont souvent employés (Hallén, 200I : 2I et plus bas). 


\section{La terminologie et classement d'emprunts lexicaux}

Dans cette deuxième partie, nous allons présenter la terminologie et les classements principaux d'emprunts lexicaux'.

\section{Terminologie}

À côté du terme "emprunt lexical ", " mot d'emprunt " ou «emprunt " (lånord), il importe de rappeler celui de "mot étranger" (främmande ord), souvent employé au sens général pour un " mot d'emprunt ", mais aussi en opposition à celui-ci, comme un stade dans le processus d'intégration et d'établissement au sein de la langue d'accueil. Les deux termes sont ainsi en opposition avec les mots " hérités ». Or, dans le cadre des recherches sur l'emprunt lexical en Suède, on rencontre le terme hemtama ord, mots "familiers ", qui peuvent être d'origine différente : empruntés, néologiques ou bien hérités (Dahlstedt, I962:31).

En effet, ces concepts sont opposés sur deux axes différents. Le " mot étranger » et le " mot familier » sont opposés sur l'axe synchronique, en prenant en compte de nombreux critères formels (prononciation, accentuation, flexion, orthographe) ou moins formels (stylistiques, sémantiques, etc.). Le "mot d'emprunt » et le "mot hérité ", eux, sont opposés sur l'axe diachronique, basé sur le critère d'origine du mot (Dahlstedt, I962 : I5). Pour cet exposé qui s'inscrit dans le cadre d'étude des influences culturelles, nous allons utiliser seulement le terme "emprunt ", étant donné son caractère de fait historique. Par conséquent, nous n'allons pas étudier les différents degrés d'intégration des mots d'origine française en suédois.

Une deuxième précision concerne la nature des mots d'emprunt : il ne s'agit pas seulement des noms communs à la base, ce qu'un « mot » d'emprunt pourrait nous amener à croire. En effet, il peut s'agir des noms propres (Sophie ou Marie en Suède) ou bien de noms communs dérivés de noms propres (majonnäs " mayonnaise ", éponyme à la base $\left.{ }^{\mathrm{IO}}\right)$. Ils peuvent aussi apparaître sous forme de sigles $\left(\right.$ laser $^{\mathrm{II}}$ ) ou bien être formés par troncation (fan "fan ", de fanatic "fanatique $\left.»^{\mathrm{I} 2}\right)$. Par ailleurs, ils ne se limitent pas aux noms, même si cette classe grammaticale reste la plus empruntable et la plus empruntée, suivie des verbes puis des adjectifs ${ }^{\mathrm{I3}}$. Toutefois, la grande majorité des exemples que nous citerons ici provient de la classe des noms. 


\section{Classement d'emprunts}

Parmi divers classements d'emprunts lexicaux, Myers-Scotton (2006 : 2I2-2I9) distingue deux catégories principales, à savoir "l'emprunt culturel » et l'emprunt de "noyau dur » ${ }^{\mathrm{I}}$. Un emprunt culturel remplit un vide dans le répertoire lexical de la langue receveuse en introduisant des nouveaux objets et concepts dans la culture de la langue emprunteuse. Un emprunt de noyau dur, en revanche, représente une " duplication » d'éléments déjà présents dans le répertoire (ibid.). L'emprunt culturel est désigné ailleurs comme "emprunt nécessaire » (behovslån), en tant que motivé par un besoin de désigner de nouveaux objets matériels, par rapport aux emprunts dits superflus, qui possèdent déjà un équivalent dans la langue d'accueil et qui font figure d' " emprunts de statut " (statuslån) en apportant « un statut plus élevé à la réalité » (Stålhammar, 20I0:22-23).

Le classement d'emprunts est généralement fondé sur l'un des trois critères suivants : l'opposition entre la forme et le sens, le degré d'assimilation à la langue d'accueil, ou bien la voie d'introduction du mot (Tournier, I998:9-I2).

\section{Emprunt de la forme et emprunt du sens}

Selon ce critère plusieurs procédés sont possibles, dont le plus courant est celui d'emprunt à la fois de la forme et du sens (l'emprunt «intégral »). C'est également à ce type que l'on fait généralement référence dans le contexte de l'emprunt lexical.

Le deuxième type, celui d'emprunt sémantique (betydelselån), implique l'emprunt du sens, ou de l'un des sens, d'un mot d'une autre langue qui se rajoute à une forme lexicale préexistante (p. ex. karaktär ${ }^{15}$ " caractère ", qui subit un élargissement de sens sous le modèle anglais en ajoutant au sens principal " ensemble de traits propres à une personne $^{\text {I6 }}$ » celui de rollfigur «personnage »; Josephson, 2004 : I 2). Dans la même catégorie se trouve le calque (översättningslån), qui représente une variante d'emprunt de sens étant donné que seul le sens est transféré tandis que la forme du mot modèle est traduite plus ou moins littéralement vers la langue emprunteuse (p. ex. tredje världen, calqué sur l'expression "tiers monde »; Edlund \& Hene, I996:34) ${ }^{17}$.

Le troisième type, l'emprunt morphologique, implique l'emprunt seulement de la forme, à laquelle on attribue un autre sens de celui que le mot possède dans la langue de sa provenance (p. ex. slip, emprunté à l'anglais par le français et utilisé au sens de « sous-vêtement masculin »; Tournier, I998 : 9). Un type particulier d'emprunt morphologique sont 
les «faux emprunts" (pseudolån, skenlån), créés avec des éléments formels issus d'une autre langue qui ne renvoient pas à un modèle direct dans la langue de laquelle proviennent les éléments (p. ex. le verbe duellera "se battre en duel ", construit en suédois sur l'emprunt duell, du fr. duel). De même, une catégorie à part est celle des emprunts " hybrides » (blandlan), avec emprunt partiel de la forme : le mot d'origine est partiellement substitué par des morphèmes de la langue d'accueil et partiellement importé (p. ex. post-it-krig ${ }^{\mathrm{I}}$, emprunté au français guerre des Post-it, avec la substitution de guerre par krig). Outre l'importation et substitution des mots, l'hybride peut figurer comme une combinaison d'un radical suédois et d'un suffixe français (läckage ${ }^{19}$ "fuite »; Dahlstedt, I962 : 22, ou bien slitage ${ }^{20}$ " abrasion »; Ståhle, I962 : 56).

\section{Degré d'assimilation}

Un deuxième classement est fondé sur le degré d'assimilation à la langue d'accueil. Ainsi un emprunt bien assimilé ne sera plus reconnaissable comme tel par un locuteur moyen (p. ex. les anciens emprunts au français tels que kalsonger "caleçon "; Dahlstedt, I962 : I9), ou bien reconnaissable comme emprunté mais considéré comme intégré (p. ex. frisör " coiffeur») ou, finalement, identifié comme étranger et non intégréer

\section{Voie d'introduction : emprunt direct et indirect}

Un emprunt lexical direct provient directement d'une langue, sans passer par une ou plusieurs langues intermédiaires ( emprunt indirect ») $)^{22}$. Ainsi de nombreux emprunts lexicaux en suédois proviennent d'une langue qui avait introduit le mot par des voies complexes. L'exemple classique est celui de pyjamas " pyjama $^{23}$ ». La voie d'introduction peut être parfois difficilement retraçable. C'est ici que l'orthographe peut se montrer utile et révéler la voie d'introduction, comme p. ex. l'emprunt nisch " niche ", d'origine française, emprunté par le suédois via l'allemand Nische ${ }^{24}$ (Edlund \& Hene, I996 : I09-I Io). Parmi les emprunts au français en suédois que nous citerons, on trouvera des mots d'origine grecque, transmis via le français, ou bien d'origine italienne.

Dans la catégorie de voie d'introduction, il importe de distinguer le « réemprunt » ou " emprunt aller-retour » (återlån), une sorte de combinaison des procédés d'emprunt direct et indirect. Il s'agit alors de réintroduire un mot dans la langue de départ en l'empruntant à celle à laquelle elle avait "prêté » le mot antérieurement. Le français a ainsi 
été la langue donneuse de vocabulaire à l'anglais qu'il a, par la suite, " réemprunté » à l'anglais. Le suédois, lui, a également emprunté ces mots à l'anglais (p. ex. biffstek, emprunté à l'anglais ${ }^{25}$, emprunté aussi par le français « bifteck»), dont le premier composant renvoie à l'ancien français boef. En français cet emprunt représente un " réemprunt partiel » (Tournier, I998:477). De même, on trouve des réemprunts intégraux à l'anglais en français, p. ex. $b_{a c o n}{ }^{26}$, emprunté par le français et par le suédois à l'anglais.

\section{L'époque actuelle}

Afin de cerner l'ampleur de l'influence du français sur le suédois aujourd'hui, nous avons, en premier lieu, analysé des listes de nouveaux mots en suédois (Nyordslistor ${ }^{27}$ ), afin de savoir sous quelle forme et par quelle voie le vocabulaire français ou d'origine française entre dans le suédois d'aujourd'hui.

En deuxième lieu, nous avons recensé des observations sur le vocabulaire d'origine française à propos des modifications qu'il subit à l'époque actuelle, suite aux influences exercées par d'autres langues, notamment de l'anglais. Nous avons ainsi testé la prononciation de quelques anciens emprunts au français afin de vérifier s'ils subissent des modifications en vue d'une meilleure adaptation à la langue suédoise (p. ex. en ce qui concerne la place de l'accent) ou bien si la prononciation a été modifiée selon le modèle de la prononciation anglaise ${ }^{28}$.

Finalement, nous avons analysé des mots d'origine française dans des secteurs sensibles à l'image de marque française, tels que la restauration et la mode, en analysant des noms qui trouvent leur inspiration dans la langue française.

\section{La nature des emprunts lexicaux français ou d'origine française en suédois à l'époque actuelle}

En consultant les listes de nouveaux mots entre 2000 et 2013 , nous avons observé diverses manières dont le français se manifeste en suédois.

Tout d'abord, nous avons relevé des emprunts à l'anglais qui sont d'origine française à la base (embrejsa " embrasser (prendre dans ses bras) ", au sens figuré " adopter ${ }^{29}$, ou triage " triage (médical) »30). Ces emprunts relèvent de l'influence française de manière indirecte, en tant qu'empruntés à l'anglais.

Ensuite, nous avons constaté que certains anciens emprunts français sont productifs $^{31}$ en suédois, probablement sur le modèle anglais 
(fiskpedikyr ${ }^{32}$ " fish pédicure ", polyamorös ${ }^{33}$ " qui a plusieurs relations amoureuses en même temps ", portföljmetoden / portfoliometoden ${ }^{34}$ méthode de "portefeuille / portfolio de compétences ", chefsnappning "séquestration de patron $\left.{ }^{35} »\right)$. Comme dans la catégorie précédente, les exemples relèvent de l'influence indirecte de la langue française. Les anciens emprunts au français font partie intégrante du lexique suédois et par conséquent procèdent à la formation des mots tout comme le lexique hérité. Leur productivité est en effet motivée par le modèle anglais.

D'autres anciens emprunts au français deviennent productifs mais sans modèle étranger direct (kalsongbadare ${ }^{36}$, ljudaffisch ${ }^{37}$ ). Parmi ces exemples nous en avons relevé un, celui de calque sur le modèle français (betårta, betårtning " entarter, entartage ${ }^{38}$ ") qui nous permet de constater l'influence indirecte du français, étant donné que les mots sont calqués sur le modèle français et non importés directement ${ }^{39}$.

Un groupe suivant contient les emprunts considérés comme provenant directement du français. L'exemple zlatanera "zlataner ${ }^{40}$ ", dérivé du prénom du footballeur suédois Zlatan Ibrahimović, a été créé par les scénaristes humoristes de l'émission Les Guignols de l'info. Citons l'exemple tiré du journal Aftonbladet (13/OI/20I3) :

(I) Med stövlar zlatanerade han isen framför båten och ytterligare jägare kunde ansluta till ön utanför Stockholm.

"Il a zlatané la glace avec ses bottes devant le bateau et d'autres chasseurs ont pu rejoindre l'île dans l'archipel de Stockholm."

D'autres exemples considérés comme emprunts directs au français sont entourage $4^{41}$ et le susmentionné post-it-krigg (< fr. " guerre des Post-it ») qui peut être aussi interprété comme emprunt hybride anglais-suédois formé sur le modèle français.

Finalement, on a relevé deux types d'influences lexicales caractérisées par un élargissement sémantique. Le premier, où d'anciens emprunts au français subissent un élargissement sémantique comme résultat de l'influence de l'anglais (karaktär " caractère ", au sens de "personnage ", cité plus haut) et le second, où les modifications sémantiques, sous l'influence de l'anglais, touchent d'anciens emprunts à l'anglais d'origine française. Citons le verbe sporta «faire du sport ${ }^{43}$ » qui acquiert le sens de " exposer, montrer, afficher ", souvent sa tenue vestimentaire, de manière voyante, selon le sens anglais du verbe to sport ${ }^{44}$. L'exemple suivant est tiré du journal Expressen (30/06/20II) :

(2) Lady Gaga sportar panda-look.

"Lady Gaga affiche un look panda". 


\section{La prononciation d'anciens emprunts au français}

Dans le cadre de cette partie de l'étude, nous avons testé la prononciation d'anciens emprunts au français afin de savoir si et dans quelle mesure elle est modifiée sous l'influence de la prononciation anglaise. Nous nous sommes inspirés des observations au sujet de l'anglification rétroactive (retroaktiv anglifiering) sur le blog linguistique Lingvistbloggen de l'Université de Stockholm en 2008 et 2009, selon lesquelles des anciens emprunts français seraient interprétés comme anglais, ce qui influencerait leur prononciation. Les mots testés étaient déjà $v u$, pose et rouge. Pour déjà vu, la prononciation sera soit "suédoise » (<deschavu: $>,<$ deschavu $>$ ) ou tendra vers la prononciation anglaise $(<$ deschavou: $>)$. Pour pose, la prononciation sera soit suédoise $(<\mathrm{po}: \mathrm{s}\rangle)$ soit " anglifiée » (<poous $>$ ), pour rouge soit suédoise ( $<$ ro:sch $>,<$ rou:sch $>$, $<$ rosch $>$ ) ou bien " anglifiée ", notamment le « $\mathrm{r} »(<\text { rou:sch }>)^{45}$. En deuxième lieu, nous avons testé la place de l'accent dans des anciens emprunts au français afin de vérifier s'ils subissent des modifications en vue d'une meilleure adaptation à la langue suédoise quant à la place de l'accent. Selon le système germanique d'accentuation, l'accent principal est situé au début du mot, contrairement aux langues romanes, où il est situé vers la fin du mot (cf. Ståhle, I962: 50-5I). Nous avons testé les adjectifs offensiv " offensif, offensive ", defensiv " défensif, défensive " et aggressiv " agressif, agressive ". La place d'accent sera soit sur l'ultième syllabe (-i'v) soit sur l'antépénultième syllabe (of'-, def'-, ag'-). Ce déplacement de l'accent dans les adjectifs en -iv commence au XX $\mathrm{X}^{\mathrm{e}}$ siècle (Dahlstedt, I962:2I). Finalement, nous avons testé la prononciation de noms qui comportent des mots qui pourraient être interprétés comme d'origine française ou anglaise afin de voir quelle prononciation prévaut ${ }^{46}$.

Nous pouvons supposer la possibilité de trois étapes dans le traitement des emprunts quant à la prononciation. Dans un premier temps, le mot préserve la prononciation rapprochée de celle de la langue d'origine (la place de l'accent aggressi'v) ${ }^{47}$. Dans un deuxième temps la prononciation est " suédifiée " (ag'gressiv, <po:s>) et finalement, " anglifiée $»(<\text { poous }>)^{48}$.

Les résultats de notre test confirment la coexistence de deux prononciations, une suédoise et l'autre " anglifiée » (déjà vu, rouge), tout comme la prononciation exclusive "anglifiée » (pose) (Figure I). En ce qui concerne la place de l'accent, l'accentuation germanique sur la première syllabe semble être bien établie (Figure 2). Finalement, les noms de type hybride (Café Continental, Ski Total) montrent une faible pro- 


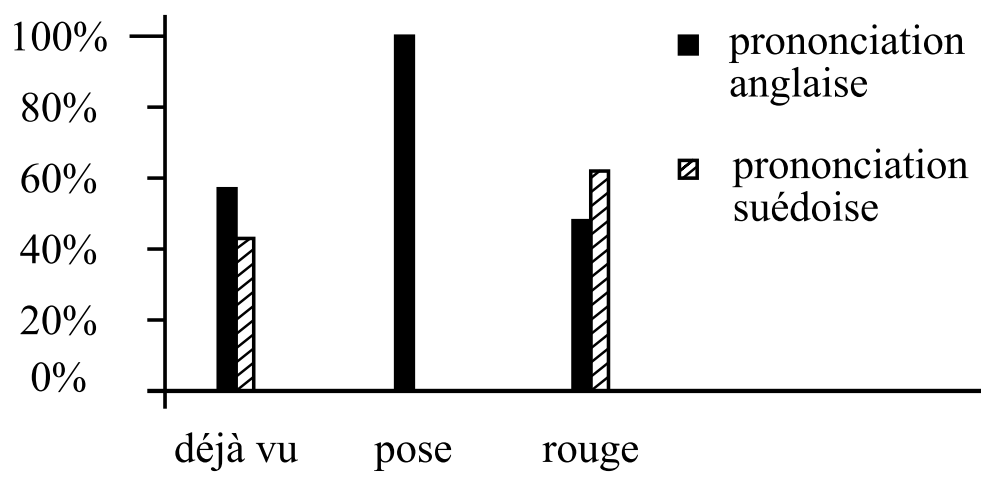

Figure 1: Prononciation de déjà vu, pose, rouge.

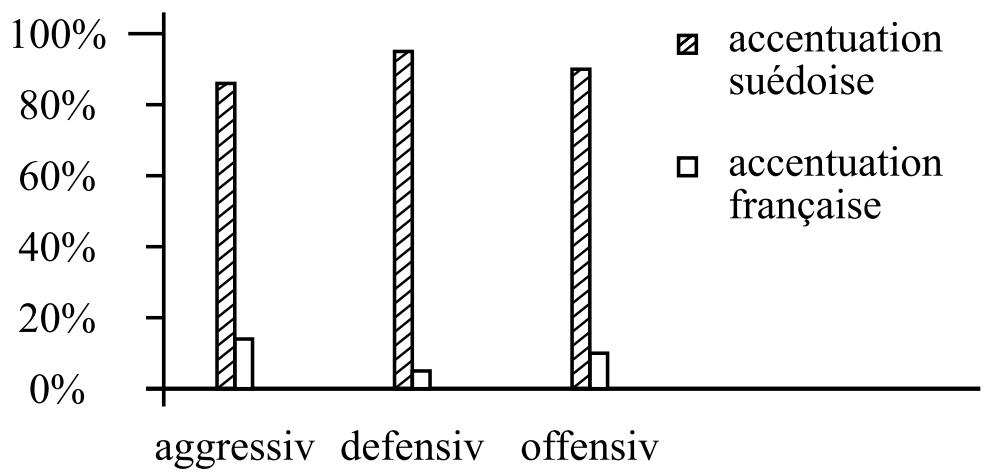

Figure 2: Accentuation d'aggressiv, defensiv, offensiv.

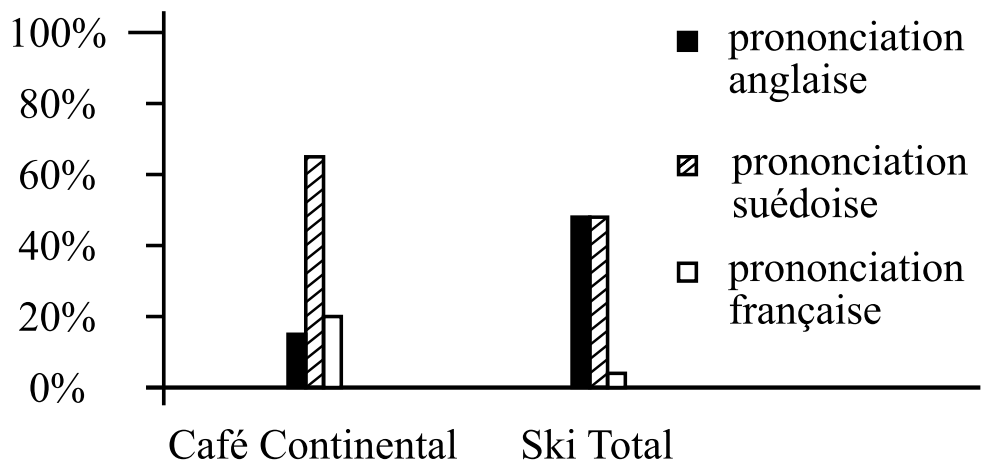

Figure 3: Prononciation de Café Continental, Ski Total.

portion de la prononciation française par rapport au suédois et à l'anglais (Figure 3). Dans l'exemple de Café Continental la prédominance de la prononciation suédoise pourrait s'expliquer par la présence du 
mot café intégré de longue date en suédois qui déclenche la prononciation suédoise du deuxième composant. Dans l'exemple Ski Total la quasi absence de la prononciation française du composant total pourrait suggérer que les locuteurs suédophones n'associent pas le mot total au français mais plutôt à l'anglais ou au suédois, tout comme le mot ski.

\section{Le prestige du français dans la Suède contemporaine}

Si le français a cédé le terrain à l'anglais en tant que fournisseur principal du suédois en mots d'emprunt, il garde de l'importance dans certains domaines de la société, comme nous l'avons déjà constaté en fin de section I. Dans ce contexte, nous avons cité Hallén (200I : 2I), qui précise que l'influence du français est notable, entre autres, dans les domaines de la restauration et de la mode en Suède.

\section{L'image de marque de la France}

Nous sommes d'avis que cette présence du français dans certains domaines de la société est étroitement liée à « l'image de marque » de la France, phénomène important de nos jours.

Dans la quête d'une image de marque, à laquelle se livrent la plupart des pays dans le monde occidental, la France semble vouloir se profiler comme porteuse de valeurs culturelles et esthétiques, tandis que les pays anglophones semblent plutôt mettre en avant une image technologique, scientifique, sportive ou économique. À titre d'exemple, considérons le numéro d'hiver 20I3 du périodique Vivre à Paris, intitulé L'excellence parisienne. Selon la publication, cette excellence s'est incarnée par « la mode, le design, les arts, la joaillerie, la gastronomie et l'artisanat de prestige ». À cet égard, on peut également regarder la liste des œuvres du Patrimoine Immatériel identifiées par l'Unesco : sur la liste des éléments de la France, on observe des phénomènes comme "Le repas gastronomique des Français ", "Le savoir-faire de la dentelle au point d'Alençon ", "La tapisserie d'Aubusson » et "La tradition du tracé dans la charpente française ", patrimoine qui semble bien se marier avec l'image de marque de la France.

Partant de ces faits, nous avons dépouillé Gula Sidorna Stockholm (Les Pages Jaunes, section de Stockholm), supposant que la présence du français serait importante dans les sphères culturelles et esthétiques, mais faible dans tout ce qui touche à la technologie et les domaines avoisinants. 


\section{Résultats et conclusions}

Conformément à l'hypothèse, nous avons trouvé peu de noms français dans les domaines technologiques, etc. Cependant, nous avons repéré 9 boutiques d'accessoires avec des noms français, Io antiquaires, I 3 boutiques de vêtements, 87 café-restaurants, et I 2 périodiques de style de vie. Nous n'avons donc pas survolé tous les domaines susceptibles de présenter une image française, mais les résultats sont à notre avis tout de même assez révélateurs, et notamment, la présence de la gastronomie française sur la liste du Patrimoine immatériel de l'Unesco semble bien fondée!

Lors du dépouillement des noms français dans la société suédoise, nous avons fait les réflexions suivantes :

- Dans les secteurs concernés, on retrouve néanmoins une majorité de noms suédois, souvent avec du "prestige implicite ", c'està-dire ayant des connotations très suédoises, loin de l'image chic offerte par le français. Ceci est le cas avec, entre autres, des établissements comme Prylboden, Samlarbolaget et Eriks bakficka.

- Contrairement à notre hypothèse, l'anglais est en effet encore plus présent dans la plupart des secteurs examinés, à l'exception du domaine de la gastronomie et de la restauration.

- Il est souvent difficile de savoir si un mot donné renvoie au français ou à l'anglais, cf. Restaurant, Café, Design, Art, Royal, Magazine, Grand.

- Il n'est parfois pas clair de savoir si une expression est un patronyme ou un nom : Androuet, Laroy, Mamadou.

- Beaucoup d'établissements portent des noms hybrides : Konditori Sans Rival, Bonne Femme Catering, À la Carte Antik.

- Il n'est pas rare de rencontrer une écriture francisante : Boutique, Compagniet, Magazine, Quriosa (!).

- Les diacritiques français sont légion : Hôtel, Complé (!)

- Les tournures de syntaxe française sont fréquentes (cf. aussi section 3 ci-dessus ${ }^{49}$ ) : Café Sodom, Brasserie Le Pot, Bistro Bohème.

\section{Conclusion}

De manière générale, le nombre d'emprunts directs au français reste faible, dans la continuité du siècle précédent. Les mots d'origine fran- 
çaise entrent notamment par le biais d'emprunt à l'anglais, langue qui reste la langue donneuse dominante de la matière lexicale en suédois aujourd'hui, témoignant de l'importance de la culture anglo-américaine à partir de la fin de la Seconde Guerre mondiale. Les anciens emprunts au français sont bien établis et productifs en suédois, même si cette productivité est souvent motivée par le modèle anglais. L'anglais influence aussi la prononciation d'anciens emprunts au français qui reçoivent une prononciation " anglifiée ». Dans le domaine commercial, le suédois demeure la langue dominante des marques commerciales en Suède. L'anglais a remplacé le français comme langue de prestige dans plusieurs secteurs, mais le français garde une certaine empreinte dans les domaines de la culture, artisanat, mode et esthétique, confirmant ainsi son image de marque. En gastronomie et restauration, le français reste plus présent que l'anglais.

\section{Notes}

I. La source de datation des emprunts utilisée est Svensk ordbok (SO), qui indique l'année de la première attestation du mot en suédois. Afin d'éviter des répétitions, cette source ne sera pas indiquée avec chaque exemple cité. Si une autre source a été utilisée, elle sera précisée avec l'exemple cité.

2. Baner est attesté depuis le milieu du XIVe siècle, d'origine germanique. Kär provient de l'anc. fr. " cher ", attesté depuis I420-I450.

3. Patron est attesté depuis I 540, du fr. patron "étalon, modèle ", une sorte d'étui à munition. Le mot furir depuis I 557 , du fr. fourrier " sous-officier chargé du cantonnement des troupes et du couchage, des distributions de vivres, de vêtements » $(\mathrm{PR})$.

4. Balkong est attesté depuis I656, de l'italien via le français; allé depuis I 663 ; assurans I626; kredit I6I7, dans le sens " confiance dans la solvabilité de qqn ", emprunté à l'italien par le français (PR); puder I650; peruk I63I ; sås I 642 ; kastrull I 690 ; ambassad I 650 , en français de l'italien, à la base un mot gotique d'origine gauloise (PR) ou bien celte $(\mathrm{SO})$; departement $\mathrm{1} 678$; porträtt I 645 ; staty I 650 . Pour d'autres exemples de mots empruntés siècle par siècle cf. Bergman (I972) ou bien Edlund \& Hene (I996).

5. Les emprunts français dans les dialectes suédois ont souvent subi des modification sémantiques. Ils peuvent avoir un sens absent en français mais présent dans le suédois standard, p. ex. blommerant " (l'air) florissant ", attesté dans les dialectes en Dalécarlie et Botnie du Nord, du fr. "bleu mourant " (bleu pâle) (Hallén, 200I : 95). D'autres emprunts ont préservé un sens vieilli ou différent de celui du suédois standard, ou montrent d'autres particularités sémantiques (ibid. : 94-98). 
6. Depuis I769. Il s'agit d'un emprunt éponyme à la base du patronyme d'Étienne de Silhouette, contrôleur général des Finances en France du milieu du XVIII siècle, qui a abandonné ses fonctions suite aux échecs de ses réformes. Par la suite, les critiques appelaient "à la silhouette » un travail non accompli (Germa I993 : 205).

7. Fåtölj est attesté depuis I 706, d'origine germanique ; garderob depuis I 729 ; kalsonger I735, d'origine italienne ; uniform I 740 ; glass I 775 ; maräng I 797 ; ridå I 787 ; pjäs I737; karikatyr I 788 ; affisch I 749 ; annons I 779 ; direktör I730; fabrik I729; populär I795; charmant I7I 6.

8. Blus est attesté depuis I 822 ; brosch I 843 ; puré, omelett et byråkrati I 822 ; charkuteri I 87 I ; socialism I 848 et diplomat I 8 I 5 .

9. De nombreux chercheurs ont observé l'imprécision du terme " emprunt ", qui est employé au sens figuré (Dahlstedt, I962 : 7). Son imprécision est due, entre autre, au fait qu'il évoque le statut des mots concernés au sein de la langue donneuse et emprunteuse (Stålhammar, 2010:22). Le terme " mot importé " ou "importation " (importord) a été introduit dans le cadre de recherches récentes sur l'emprunt lexical dans les pays nordiques. Le terme « emprunt » reste néanmoins le plus établi au niveau mondial.

Io. D’après mahonnaise, de Port-Mahon, en souvenir de la prise de ville par Richelieu, ou bien d'après la variante d'orthographe de " bayonnaise " (Germa, I993 : I47). En suédois depuis I 883.

I I. Emprunté à l'anglais light amplification by stimulated emission of radiation.

I2. En français depuis I909, " admirateur ou admiratrice enthousiaste d'une vedette ", réemprunt partiel à l'anglais (Tournier, I998 : I65). En suédois depuis I95 I, emprunté à l'anglais.

I3. Ou des adjectifs et des verbes. Des recherches montrent que verbes et adjectifs empruntés dans un corpus déterminé sont souvent proches en nombre, p. ex. dans le corpus de Chrystal (I988: 77) dans le cadre de la recherche sur des emprunts anglais en suédois.

\section{I4. Cultural borrowings et core borrowings.}

I 5. Attesté en suédois depuis I755, emprunté au français caractère au sens de " lettre; ensemble de signes distinctifs ».

I6. " sammanfattningen av de inre egenskaper som är kännetecknande för en person » $(\mathrm{SO})$.

I7. Edlund \& Hene (I996:33-35) englobent l'emprunt sémantique (betydelselån) et le calque (översättningslån) dans la catégorie begreppslån, " emprunt de concept ». L'expression " tiers monde »(tredje världen) paraît dans les années I950. 
I 8. Nouveau mot en suédois en 20I I (NL 20II).

I9. Attesté depuis I749.

20. Depuis 1697 .

2 I. À ce propos il importe de mentionner le "xénisme ", un mot étranger qui évoque souvent la culture étrangère, et le " pérégrinisme », un emprunt de passage qui ne reste employé que pour une circonstance particulière (cf. Tournier, I998 : IO).

22. Les termes " emprunt direct " et " indirect "sont employés également dans un autre sens. Ljung (I988) fait la distinction entre emprunts directs (direkta lån) par opposition aux calques (översättningslån) et emprunts structuraux (konstruktionslån) où l'influence est moins directe en tant qu'elle est exercée sur la syntaxe, la flexion ou la formation des mots (emprunts structuraux). La langue d'accueil peut ainsi procéder à une traduction littérale du mot en question (calques). L'emprunt direct, en revanche, implique « la transposition directe d'un morphème étranger » ( "den direkta överföringen av främmande morfem »; Chrystal, ı988: І3).

23. En suédois emprunté à l'anglais, attesté depuis I903. Par l'intermédiaire de l'hindi, de l'origine perse.

24. En suédois depuis I69I.

25. De l'anglais beefsteak, depuis I 765 .

26. Emprunté à l'anglais par le français, attesté depuis I 834, de l'anc. fr. bacon " lard maigre salé » (Tournier, I998:477). En suédois depuis I924.

27. Les listes sont publiées chaque année par le Conseil des langues de Suède (Institutet för språk och folkminnen) sur le site du Conseil et dans le magazine linguistique Språktidningen. Le recueil des mots est fait sur des corpus des journaux quotidiens et d'autres médias. Le Conseil reçoit également des propositions individuelles de personnes extérieures.

28. 2 I informants ont participé au test, dont I 6 âgés de I7 ans et 5 âgés de I 8 ans. Ils sont tous locuteurs natifs du suédois. Ils ont passé un an en France à l'École suédoise du lycée Saint Dominique de Neuilly-sur-Seine et ont des connaissances du français. La tâche consistait à lire des phrases de textes en suédois qui contenaient des emprunts français. Les phrases provenaient de blogs divers recueillis sur internet (voir annexe).

29. Attesté depuis 2006, son utilisation augmente en 2013 ; d'après l'anglais embrace (NL 2013).

30. Depuis 2006, du français via l'anglais (NL 2006). 
3I. Dans le sens qu'ils participent à la création des mots composés et par conséquent à l'élargissement du lexique.

32. Pédicure qui utilise des poissons. Nouveau mot en 2009 (NL 2009; la langue source n'est pas indiquée), avec l'ancien emprunt pedikyr " pédicure » (depuis I 899).

33. Nouveau mot en 2004, sur le modèle anglais polyamourous, avec l'emprunt amorös "érotique " du fr. "amoureux " (attesté depuis I9I3) et le préfixe grec poly- « multi-, pluri- » (NL 2004; cf. aussi le substantif polyamori "le fait d'avoir plusieurs relations amoureuses en même temps »).

34. Attesté depuis 1997, nouveau mot en 2000 (NL 2000), avec l'ancien emprunt portfölj " portefeuille » ( 1758$)$, fait vraisemblablement sur le modèle anglais portfolio method.

35. Nouveau mot en 2009, d'après le terme anglais bossnapping, utilisé en France (NL 2009), avec l'ancien emprunt chef (I727).

36. " personne qui se baigne en portant son slip de marque sous son caleçon de bain faisant en sorte que la marque soit bien visible » (NL 2008); mot composé avec l'élément kalsong, du fr. caleçon (I735).

37. Une sorte de poster sonore. Nouveau mot en 2008 (NL 2008), crée avec l'emprunt affisch " affiche " (I749).

38. Nouveaux mots en 200I, employés dans le contexte de " attentat pâtissier » (NL 200I), construits avec l'ancien emprunt tårta (attesté depuis I 555 , introduit via le bas-allemand du français " tarte ") mais en même temps calqué sur le modèle français " entarter, entartage ".

39. Cf. la note 22.

40. Nouveau mot en 2012 (NL 20I2), au sens de " réussir quelque chose avec force; dominer ».

4I. Attesté depuis I993. Nouveau mot en 2007, au sens d'entourage d'une personnalité célèbre (NL 2007). Étant donné un emploi identique en anglais, son influence n'est pas exclue.

42. NL 20II. Il s'agit d'une pratique qui consiste à décorer les vitres d'une entreprise avec des Post-it de différentes couleurs. Notons ici le mot français " post-it ", emprunté à l'anglais (I985; Tournier I998: 564).

43. Attesté depuis I 885 ; cf. sport, emprunté à l'anglais vers I 857 mais d'origine française au départ.

44. NL 2009.

45. Pour la présentation de la prononciation nous n'avons pas utilisé l'alpha- 
bet phonétique international mais plutôt une transcription adaptée de façon approximative à la prononciation française.

46. Les noms testés étaient Café Continental et Ski Total, dont la prononciation de « continental » et « total » peut se rapprocher soit de la prononciation française, soit anglaise ou encore suédoise.

47. La prononciation <po:z> reste hypothétique car le son « $\mathrm{z} »$ français n'existe pas en suédois.

48. Nous n'avons pas observé le déplacement de la place de l'accent vers celle que les adjectifs testés ont en anglais, à savoir sur la pénultième syllabe.

49. Dans les exemples cités suivants le mot principal se trouve à gauche (Bistro Bohème au lieu de Bohème Bistro), comme dans Café Continental et Ski Total.

\section{Bibliographie}

Andersson, H. (1999), Vokabler på vandring. Ordimport till Sverige under tusen ar. Lund : Studentlitteratur.

Bergman, G. (1972) [1968], Kortfattad svensk språkhistoria. En översikt över det svenska språkets utveckling från de äldsta nordiska runinskrifterna fram till vår egen tid. Stockholm : Prisma.

Chrystal, J.-A. (1988), Engelskan i svensk dagspress. [Skrifter utgivna av Svenska språknämnden 74]. Stockholm : Esselte Studium.

Dahlstedt, K.-H. (I962), «Vad är främmande ord? » in Dahlstedt, K.-H., Bergman, G. \& Ståhle, C.I., Främmande ord i nusvenskan. Verdandis skriftserie I7. Stockholm : Svenska bokförlaget, Bonniers : I-3 I.

Edlund, L.-E. \& Hene, B. (I996) [I992], Lånord i svenskan. Om språkförändringar $i$ tid och rum. Stockholm : Norstedts.

Engwall, G. (1994), "Svenskt och franskt " in Arv och lån i svenskan. Sju uppsatser om ordförrådet $i$ kulturströmmarnas perspektiv. Stockholm : Svenska Akademien \& Norstedts : 5 I-63.

Germa, P. (I993), Du nom propre au nom commun, dictionnaire des éponymes. Paris : Bonneton.

Hallén, K. (200I), Franskt $i$ svensk tappning. Studier över franska lånord $i$ svenska dialekter, thèse de doctorat [partie I]. Uppsala : Skrifter utgivna av Språk- och Folkminnesinstitutet, Dialektavdelningen A : 28.

Josephson, O. (2004), Ju. Ifrågasatta självklarheter om svenskan, engelskan och alla andra språk $i$ Sverige. Stockholm : Norstedts. 
Le nouveau Petit Robert de la langue française (PR) (2009). Paris : Le Robert.

Ljung, M. (I988), Skinheads, hackers \& lama ankor. Engelskan i 8o-talets svenska. Stockholm : Trevi.

Myers-Scotton, C. (2006), Multiple Voices. An Introduction to Bilingualism. Malden, MA : Blackwell Publishing.

Ståhle, C.I. ( I962), « De främmande orden och den svenska ordbildningen » in Dahlstedt, K-H., Bergman, G. \& Ståhle, C.I., Främmande ord i nusvenskan. Verdandis skriftserie I7. Stockholm : Svenska bokförlaget, Bonniers : $48-73$.

Stålhammar, M. (2010), Engelskan i svenskan. Åttahundra år av lånade ord och språkliga influenser. Stockholm : Norstedts.

Svensk ordbok utgiven av Svenska Akademien (SO) (2009). Stockholm : Svenska Akademien.

Tournier, J. (I998), Les mots anglais du français. Paris : Belin.

Vivre à Paris, numéro d'hiver 2013.

\section{Références internet}

Gula Sidorna : http://gulasidorna.eniro.se/hitta:gula+sidorna/stockholm.

Lingvistbloggen : http://lingvistbloggen.ling.su.se/.

Nyordslistan (NL) 2000-20I3 : http:/www.sprakochfolkminnen.se/sprak/ nyord/nyordslistor.html.

Patrimoine Immatériel de la France, Unesco : http://www.unesco.org/culture/ ich/index.php? $\lg =$ fr $\& p g=003$ I I \& topic $=m p \& c p=F R$.

\section{Annexe}

Phrases contenant les mots d'emprunt testés auprès des informants suédophones.

Jag får ofta deja vu-känslan. Det känns som man gjort något förut. Jag vet inte riktigt varför man får deja vu. Jag fick en riktigt ordentligt deja vu-upplevelse i morse när jag la på deodorant.

Minns när jag var I4 och stod och posade framför spegeln. Vi har alla vår « spegel-pose ». Det är inte samma sak att posa framför en kamera 
som framför en spegel. Spegel-posen är helt undermedveten och sker utan att vi tänker på det. Vi ser vår pose bara hemma i badrummet.

Man kan skriva hur mycket som helst om highlighters och rouge som man kan använda och få till riktigt bra effekter om man vet vad man sysslar med. Det finns flera olika former av rouge, men själv använder jag puderrouge som jag applicerar med en rougeborste. Jag kör en klassiker och formar munnen som ett « $\mathrm{O}$ » och lägger rouget precis under och i höjd med kindbenet.

Det finns olika spelstilar som beror av hur aggressivt man spelar. Det finns offensivt och defensivt spel så man kan spela mer offensiv eller defensiv fotboll till exempel.

I tio års tid har Café Continental bjudit på musik från hela världen varje vecka.

Jag har köpt en snowboard och boots på Ski Total i Uppsala. 